\title{
Do bulges stop stars forming?
}

\author{
Stephen Eales, ${ }^{1,2 \star}$ Oliver Eales ${ }^{3}$ and Pieter de Vis ${ }^{1}$ \\ ${ }^{1}$ School of Physics and Astronomy, Cardiff University, The Parade, Cardiff CF24 3AA, UK \\ ${ }^{2}$ Institute of Astronomy, University of Cambridge, Madingley Road, Cambridge CB3 OHA, UK \\ ${ }^{3}$ Department of Infectious Disease Epidemiology, School of Public Health, Faculty of Medicine, Imperial College London, Medical School Building, St \\ Mary's Campus, Norfolk Place, London W2 1PG, UK
}

Accepted 2019 September 26. Received 2019 September 20; in original form 2019 June 25

\begin{abstract}
In this paper, we use the Herschel Reference Survey to make a direct test of the hypothesis that the growth of a stellar bulge leads to a reduction in the star formation efficiency of a galaxy (or conversely a growth in the gas-depletion time-scale) as a result of the stabilization of the gaseous disc by the gravitational field of the bulge. We find a strong correlation between star formation efficiency and specific star formation rate in galaxies without prominent bulges and in galaxies of the same morphological type, showing that there must be some other process besides the growth of a bulge that reduces the star formation efficiency in galaxies. However, we also find that galaxies with more prominent bulges (Hubble types E to Sab) do have significantly lower star formation efficiencies than galaxies with later morphological types, which is at least consistent with the hypothesis that the growth of a bulge leads to the reduction in the star formation efficiency. The answer to the question in the title is therefore yes and no: bulges may reduce the star formation efficiency in galaxies but there must also be some other process at work. We also find that there is a significant but small difference in the star formation efficiencies of galaxies with and without bars, in the sense that galaxies with bars have slightly higher star formation efficiencies.
\end{abstract}

Key words: galaxies: evolution.

\section{INTRODUCTION}

When investigating galaxy evolution astronomers have the big advantage over real historians or archaeologists that they can see galaxies in the past. This sometimes seems a rather tantalizing advantage because we can only see a galaxy at one moment in time and cannot watch the evolution of an individual galaxy. In practice, we are forced to adopt a statistical approach. The first step in this approach is the uncontroversial, although challenging, one of building up, through observational programmes, a statistical description of the galaxy population at each cosmic epoch. The second step, however, is subject to - and actually requires - theoretical bias because it involves inferring the physical connections between the galaxy populations at the different epochs.

For example, suppose that there is a class of galaxies at $\mathrm{z}=1$ that has very similar properties to a class of galaxies at $\mathrm{z}=0$. To give a concrete example, let us imagine that the low-redshift and high-redshift classes both contain galaxies with an exponential stellar disc. Does this similarity imply that the galaxies in these classes have not evolved very much? The answer here is obviously no because it is perfectly possible that a galaxy with an exponential disc at $\mathrm{z}=1$ might now have a very different stellar structure, and a galaxy with an exponential disc at $z=0$ might have had a very different structure at a $z=1$. Inferring the physical connections is not only challenging but is actually impossible without some prior theoretical assumptions about how galaxies evolve. On top of this rather abstract problem, there is also the very practical problem that many of the basic physical properties of galaxies are remarkably difficult to measure. For example, there are not many properties more basic than the star formation rate (SFR) in a galaxy, but there are at least 12 different methods that have been proposed for measuring the SFR, none of which is entirely satisfactory (Kennicutt \& Evans 2012; Davies et al. 2016).

In view of the difficulty in measuring such basic physical properties, much of the work in the field over the last few decades has been based on statistical descriptions using simple observational parameters. The classic example is the diagrams of optical colour versus absolute magnitude plotted for galaxies found in large optical surveys. The galaxies found in these surveys fall in two main areas of these diagrams: a narrow band occupied by galaxies with red colours called the 'red sequence' and a more extended region of galaxies with blue colours, the 'blue cloud', separated by the 'green valley' where there are fewer galaxies (Baum 1959; Strateva et al. 2001; Bell et al. 2003; Baldry et al. 2012). The red sequence is mostly but not completely (Cortese 2012, and references therein) 
composed of galaxies with morphologies in the early-type class (ellipticals and S0s), whereas the blue cloud is mostly composed of late-type galaxies (LTGs; irregulars or disc-dominated galaxies). This simple observational picture has given rise to the natural conclusion that there are two distinct classes of galaxy: star-forming galaxies in the blue cloud and galaxies in which there is now very little star formation - variously called 'red and dead', 'quiescent', or 'quenched' galaxies - forming the red sequence. A consequence of this dichotomy is that there must be some physical process that converts a star-forming galaxy in the blue cloud into a galaxy with very little star formation on the red sequence, and this process must have quenched the star formation quickly (at least quickly relative to the age of the Universe) to explain the relative dearth of galaxies in the green valley, although there is no consensus about the identity of this quenching process (Eales et al. 2018a, and references therein).

In a series of three papers, we have argued for an alternative model of galaxy evolution. A hint that the picture above is not correct is that the galaxies found in a submillimetre survey do not lie on a colour-absolute-magnitude diagram in the classic blue cloud and red sequence but instead have almost the opposite distribution, forming a 'green mountain' (Eales et al. 2018b). This result suggests, at least, that the current picture of galaxy evolution may be an accident of the fact that our knowledge of the galaxy population has hitherto been almost entirely based on the galaxies found in optical surveys.

In the first paper in the series, we used the Herschel Reference Survey (HRS) to show that on a diagram of specific SFR (SFR divided by stellar mass; sSFR) versus stellar mass - meaningful physical properties of galaxies rather than simple colours and absolute magnitudes - galaxies lie on a single curved Galaxy Sequence (GS) rather than the two separate distributions of star-forming and red-and-dead galaxies suggested by the current paradigm (Eales et al. 2017). The morphologies of galaxies change gradually along the GS rather than there being an obvious break between LTGs and early-type galaxies (ETGs; Eales et al. 2017). In another paper, we showed that a single continuous GS in this plot of sSFR versus stellar mass naturally leads to the distinctive distributions in the colourabsolute-magnitude space as a result of the different selection effects operating in the optical and submillimetre wavebands (Eales et al. 2018b). This picture of a single GS is also consistent with recent studies that show the kinematic properties of galaxies appear to change gradually along the Hubble sequence rather than there being an abrupt change at the early-type/late-type boundary (Cappellari et al. 2013; Cortese et al. 2016).

The single GS, and the gradual variation in galaxy properties along it, does not require a rapid quenching process, in contrast to the standard picture of a 'star-forming main sequence' and separate region of red-and-dead galaxies. We showed that the curvature of the GS and the rapid low-redshift evolution of the galaxy population seen in radio and submillimetre surveys can be produced by a weak quenching process in which the gas supply to galaxies is stochastically turned off, with the curvature of the GS and the evolution being produced as the galaxies gradually use up their remaining gas (Eales et al. 2018a).

Another galaxy property that also seems to vary gradually along the GS is star formation efficiency (SFR divided by gas mass; SFE) or its reciprocal, the depletion time (gas mass divided by SFR). A large number of studies, both of galaxies at low redshift (Saintonge et al. 2011, 2012, 2017; Eales et al. 2018a) and high redshift (Scoville et al. 2017; Tacconi et al. 2018) have found that SFE and sSFR are strongly correlated, with galaxies with lower values of sSFR also having lower values of SFE. There is no consensus about the physical cause of this correlation but one appealing possibility is that it is connected to the morphological change along the GS. Martig et al. (2009) have suggested that the SFE will be less in a galaxy with a large bulge-to-disc ratio, since the gas in the galaxy's disc will be stabilized against collapse into stars by the gravitational field of the bulge (see fig. 3 of that paper). A corollary of that work is that the decrease in SFE at low values of SSFR follows from the fact that galaxies with low values of SSFR also tend to have more prominent bulges.

There is some indirect observational evidence for this hypothesis. Saintonge et al. (2012) showed that galaxies in the GASS and COLD GASS surveys that have low values of SFE also have high values of the SDSS concentration index and central surface density, both of which are characteristic of galaxies with high bulge-to-disc ratios. Genzel et al. (2014) have used high-resolution observations of $\mathrm{H} \alpha$ to show that in the central regions of high-redshift galaxies the SFR is often lower than in the surrounding regions, the surface density is high, and the gas is stable against gravitational collapse - all of which is consistent with the hypothesis that the growth of a central bulge in these galaxies has stabilized the gas and reduced the SFE.

This paper describes a more direct attempt to test this hypothesis. The studies of the relationship between SFE and sSFR cited above all used samples of galaxies that are too distant for the morphologies of the galaxies to be classified. The HRS, however, contains galaxies that are close enough for the morphologies of almost all of the galaxies to be known (Boselli et al. 2010). By using the HRS galaxies, we can make a simple test of the hypothesis that the reduction in the SFE in a galaxy is entirely the result of the growth of a bulge.

We assume a Hubble constant of $67.3 \mathrm{~km} \mathrm{~s}^{-1} \mathrm{Mpc}^{-1}$ and the other Planck cosmological parameters (Planck Collaboration 2014).

\section{METHODS}

\subsection{The Herschel reference survey}

The sample we use in this paper is the HRS (Boselli et al. 2010). The HRS consists of 323 galaxies with distances between 15 and $25 \mathrm{Mpc}$ and with a near-infrared $K$-band limit of $K<8.7$ for ETGs (E, SO, and SOa) and $K<12$ for LTGs (Sa-Sd-Im-BCD). The sample was designed to select most of the galaxies above stellar mass limits (different for ETGs and LTGs) in a given volume of space for an observing programme with the Herschel Space Observatory (Pilbratt et al. 2010). The different $K$-band limits for ETGs and LTGs were chosen to avoid the sample being dominated by lowmass ETGs, which would have been hard to detect with Herschel. Eales et al. (2017) estimate that the HRS contains all LTGs in the HRS volume with stellar masses above $\simeq 8 \times 10^{8} \mathrm{M}_{\odot}$ and all ETGs above $\simeq 2 \times 10^{10} \mathrm{M}_{\odot}$. Despite the high-mass limit for ETGs, Eales et al. also show that $\simeq 90$ per cent of the stellar mass in this volume in ETGs with stellar masses $>10^{8} \mathrm{M}_{\odot}$ is contained in the galaxies included in the HRS. The reason for this low value is the turnover in the stellar mass function for ETGs (Baldry et al. 2012), which means that although ETGs with low stellar masses are missing from the HRS, they actually contain only a small percentage of the total stellar mass in the ETG class. The HRS therefore contains most of the stellar mass in a given volume of space, and the size of this volume means that it is a fair sample of what has been produced by 12 billion years of galaxy evolution, with the only caveat being that the volume contains the Virgo cluster and so cluster galaxies may be over-represented.

For the purpose of this paper, we need to be able to estimate the mass of the interstellar medium in each HRS galaxy. Although 
many of the HRS galaxies have been observed in the CO 1-0 and 21-cm lines, the standard methods of estimating the mass of the molecular and atomic phases of the ISM, only about 70 per cent of the HRS galaxies have been observed in the CO 1-0 line (Boselli, Cortese \& Boquien 2014).

Fortunately, many authors have argued that estimating the mass of the ISM in a galaxy from the continuum dust emission is actually better than the standard method (e.g. Eales et al. 2012; Magdis et al. 2012; Scoville et al. 2014). The main advantages are (1) the uncertainty in the $\mathrm{X}$ factor used to connect the intensity of the optically thick CO 1-0 line to the column density of molecular hydrogen, (2) the evidence from Fermi (Abdo et al. 2010), Herschel (Pineda et al. 2013), and Planck (Planck Collaboration XIX 2011) that one-third of the molecular gas in our galaxy is not emitting $\mathrm{CO}$, (3) the evidence for a much simpler relationship between metallity and the dust-to-gas ratio than for the CO X factor (Sandstrom et al. 2013; Rémy-Ruyer et al. 2014). For the HRS, we are much better placed to use this alternative method because all the HRS galaxies were observed with Herschel in five far-infrared or submillimetre bands (Ciesla et al. 2012; Smith et al. 2012; Cortese et al. 2014). Of the 323 HRS galaxies, 284 ( 88 per cent) were detected in at least one band. In this paper, we adopt this alternative method of estimating the mass of the ISM.

\subsection{Star formation rates, stellar masses, and dust masses}

The HRS galaxies have high-quality photometry in 21 photometric bands from the $U V$ to the submillimetre (Eales et al. 2017 and references therein), making it ideal for the application of a galaxy modelling program such as MAGPHYS (Da Cunha, Charlot \& Elbaz 2008). De Vis et al. (2017) applied MAGPHYS to the HRS galaxies, and we use their estimates of SFRs, stellar masses, and dust masses in this paper. We refer the reader to that paper for a detailed description of the application of MAGPHYS to the HRS.

Very briefly, MAGPHYS generates 50000 possible models of the spectral energy distribution (SED) of the unobscured stellar population, based on the stellar synthesis models of Bruzual \& Charlot (2003), and 50000 models of the dust emission from the ISM, based on the ISM model of Charlot \& Fall (2000). The two sets of models are linked by balancing the energy absorbed by the dust in the optical and near-infrared with the energy emitted from the dust in the mid-infrared to submm wavebands, which generates a large number of possible SEDs. These are then fitted to the photometric measurements. For our project, an advantage of MAGPHYS is that it generates a probability distribution for each galaxy property - in our case SFR, stellar mass, and dust mass - from the quality of the fits between the SEDs and the measurements.

We showed in Eales et al. (2017) that there is good agreement between the stellar masses produced by MAGPHYS and stellar masses estimated using a more empirical method. We show here that there is also good agreement between the MAGPHYS estimates of dust mass and empirical estimates. Fig. 1 shows a comparison of the MAGPHYS estimates with the estimates of dust mass made by Cortese et al. (2014) for the 204 HRS galaxies that have detections in all five Herschel bands, using fits of modified blackbodies to the Herschel photometry. We have corrected the latter estimates to allow for the different Hubble constant used in this paper and for the dust-mass opacity coefficient used in MAGPHYS. The agreement is extremely good with the best-fitting straight line having a close to unity slope $\left(\log _{10} \mathrm{M}_{\mathrm{d}, \mathrm{C} 14}=0.979 \log _{10} \mathrm{M}_{\mathrm{d}, \mathrm{MAGPHYS}}+1.91 \times 10^{-5}\right)$ and a root-mean-squared variation around the line of only 0.067 .

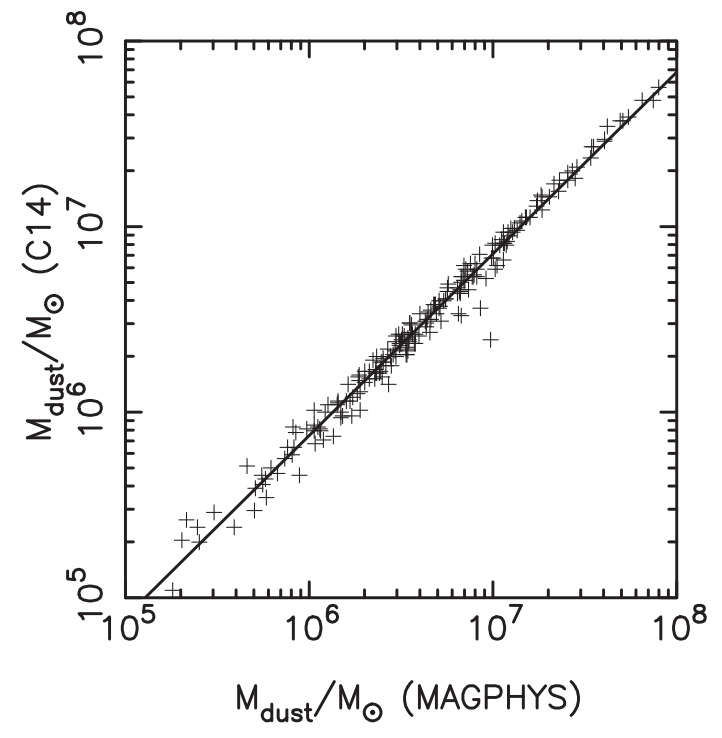

Figure 1. Dust mass estimated by MAGPHYS versus dust mass estimated by Cortese et al. (2014; C14) using a single-temperature modified blackbody for the 204 HRS galaxies with detections in all five Herschel bands. The C14 dust masses have been corrected to the Hubble constant used in this paper and to the dust-mass opacity coefficient used in MAGPHYS. The line is the best-fitting straight line and has the form $\log _{10} \mathrm{M}_{\mathrm{d}, \mathrm{Cl} 14}=$ $0.979 \log _{10} \mathrm{M}_{\mathrm{d}, \text { MAGPHYS }}+1.91 \times 10^{-5}$. The root-mean-squared variation around the line in the $y$-direction is 0.067 .

It is not possible to test the MAGPHYS estimates of the SFR in the same way because there is no consensus about the best empirical method of estimating the SFR. A major advantage of MAGPHYS over most empirical methods (Kennicutt \& Evans 2012; Davies et al. 2016) is that it avoids the problem for optically based methods of missing the star formation that is occurring deep in molecular clouds. One obvious disadvantage is that it is based on the assumption that galaxies are isotropic, but Hayward \& Smith (2015) have used simulated galaxies to investigate the effect of viewing angle, finding that when a simulated disc galaxy is viewed from different directions the variation in the MAGPHYS estimates of the SFR is mostly within the $\pm 1 \sigma$ error range estimated by MAGPHYS. Davies et al. (2016) also did an extensive comparison of the different methods that have been used for estimating SFRs, using 12 different methods to estimate the SFRs for 4000 starforming galaxies. As their gold standard, they used the SFRs estimated from the method of Popescu et al. (2011), which takes into account the individual geometry of each galaxy. They found that the slope of the relationship between SFR and stellar mass found by this method and by MAGPHYS were very similar.

\subsection{Gas masses}

We estimated the masses of gas in the HRS galaxies from the continuum dust emission using two alternative approaches.

A number of groups have calibrated the relationship between the luminosity of the continuum dust emission and the gas masses determined from the standard CO 1-0 and 21-cm method (Eales et al. 2012; Scoville et al. 2014; Groves et al. 2015; Janowiecki et al. 2018). An advantage of this method, rather than going through the intermediate step of calculating the dust mass, is that it avoids the need to know the value of the dust-mass opacity coefficient or the gas-to-dust ratio, neither of which are known accurately (Clark et al. 
2016). A disadvantage of the method is that it suffers from the same limitations of the $\mathrm{CO} / 21-\mathrm{cm}$ method; if, for example, some fraction of the molecular gas does not contain any $\mathrm{CO}$, the dust method will also miss this gas.

Since Janowiecki et al. (2018) calibrated their relationship from 68 galaxies in the HRS, we have used their relationship between the mass of molecular gas and the 500- $\mu$ m luminosity:

$\log _{10}\left(\frac{M_{H_{2}}}{\mathrm{M}_{\odot}}\right)=0.99\left(\log _{10} \frac{L_{500 \mu m}}{\mathrm{~L}_{\odot}}-9.0\right)+8.41$.

This method, of course, will produce an estimate of the mass of gas in the molecular phase rather than the total mass of the ISM.

The second method we use was originally suggested by James et al. (2002) but was recently updated by Clark et al. (2016). The method is based on the assumption, for which there is a lot of observational support (Clark et al. 2016 and references therein), that a constant fraction of the metals in a galaxy are in dust grains. If this is the case, the mass of gas in a galaxy is given by

$M_{\mathrm{g}}=\frac{M_{\mathrm{d}}}{\epsilon_{\mathrm{d}} f_{Z_{\odot}} Z}$,

where $\epsilon_{\mathrm{d}}$ is the fraction of the mass of metals in a galaxy that is incorporated in dust grains, $f_{Z_{\odot}}$ is the metal mass fraction at solar metallicity, and $Z$ is the metallicity of the ISM in a galaxy as a fraction of the solar value. We have assumed that the value of $\epsilon_{\mathrm{d}}$ is 0.5 (Clark et al. 2016) and a value for $f_{Z_{\odot}}$ of 0.0142 . We have used the metallicities measured for HRS galaxies by Hughes et al. (2013). For the HRS galaxies without metallicity measurements, which are mostly ETGs, we estimated the metallicity of the galaxy using the relationship between metallicity and stellar mass derived by Hughes et al. (their equation 5). We have used the estimates of dust mass produced by MAGPHYS.

A disadvantage of this method is that it relies on the value of the dust-mass opacity coefficient used in MAGPHYS being correct. Errors in this, and in any of the constants in equation (2), will merely lead to all the gas masses being wrong by the same factor, and will not lead to a spurious relationship between SFE and SSFR.

\section{RESULTS}

Fig. 2 contains four panels. The two upper panels show the ratio of gas mass to stellar mass plotted against sSFR. The two bottom panels show SFE plotted against sSFR. In all panels, we have calculated the gas mass using equation (1). The panels on the left of Fig. 2 are for LTGs and the panels on the right are for ETGs. However, in this figure we have redefined the two classes. In Fig. 2, the ETGs are all those galaxies with a prominent bulge, which we have defined as the morphological types on the Hubble sequence between, and including, Sbc and E (121 galaxies). The LTGs are all galaxies with morphological types Sc or later (118 galaxies). Fig. 3 shows the same four panels but this time with the gas mass calculated using equation (2). In both figures, the dashed black lines in each panel show the best fits of the equation $\log _{10} y=A \log _{10} x+B$ to the data. The values of $A$ and $B$ are given in Table 1, as are the values of the Spearman correlation coefficient for each data set.

Let us consider first the top panels of Figs 2 and 3, the plots of the ratio of gas mass to stellar mass versus sSFR. The beige points in both panels show the average values of this ratio for the molecular gas for galaxies in the xCOLD GASS survey (Saintonge et al. 2017), which were not separated by morphological type. In Fig. 2, the agreement is good for both ETGs and LTGs. In Fig. 3, the points from xCOLD GASS are significantly lower than our points, although we see the same trend between the mass ratio and SSFR. Apart from the possibility that we are using the wrong values for the constants in equation (2), the obvious explanation of the discrepancy is that in Fig. 3 we are using estimates of the entire mass of the ISM not just the molecular phase.

Now, let us consider the bottom panels of both figures, the plots of SFE versus sSFR. The blue-dashed line in Fig. 2 shows the relationship found for the galaxies in xCOLD GASS (Saintonge et al. 2017) and the red-dashed line shows the relationship for zero redshift found by Scoville et al. (2017). We have not plotted these relationships in Fig. 3 because they only include the molecular phase of the ISM. Strong relationships between SFE and sSFR are seen in our data in both Figs 2 and 3, for both the ETGs and LTGs, with slopes similar to those found in the other studies, although there are significant offsets (Table 1), which it seems likely are caused by systematic differences in the methods used to estimate gas mass and SFR.

At this point, it is important to consider whether all the relationships might be spurious. Both SFE and sSFR are ratios with SFR as the numerator. Errors in the SFR might therefore produce a spurious correlation between SFE and sSFR, a possibility that, of course, applies to all previous studies of this sort. Appendix A describes an investigation of this possibility. We show there that the errors do produce a correlation between SFE and SSFR but with a much shallower slope than that seen in Figs 2 and 3.

We further investigated the relationships between the different variables using the Metropolis-Hastings algorithm to fit the following two equations to the data (Appendix B). Following Scoville et al. (2017), these relationships allow for the possibility that stellar mass is an important variable.

$$
\begin{aligned}
& M_{\mathrm{gas}}=A \mathrm{M}_{\odot} \times\left(\frac{\mathrm{sSFR}}{10^{-10}}\right)^{B} \times\left(\frac{M_{*}}{10^{10} \mathrm{M}_{\odot}}\right)^{C}, \\
& \mathrm{SFR}=A \mathrm{M}_{\odot} \mathrm{yr}^{-1} \times \frac{M_{\mathrm{gas}}}{10^{9} \mathrm{M}_{\odot}} \times\left(\frac{\mathrm{sSFR}}{10^{-10}}\right)^{B} \times\left(\frac{M_{*}}{10^{10} \mathrm{M}_{\odot}}\right)^{C} .
\end{aligned}
$$

The results are given in Table 2. The values derived for B in the second equation are reassuringly similar to the values for the slope of the relationship between SFE and SSFR given in Table 1.

\section{DISCUSSION}

The correlations between SFE and specific star formation rate (sSFR) are much stronger in Fig. 2 than Fig. 3. Since the gas masses used in Fig. 2 only include molecular gas, whereas the gas masses in Fig. 3 incorporate all phases of the ISM in which dust is found, the tighter correlation in Fig. 2 is easily explained by the fact that stars form out of molecular gas.

The most interesing result apparent in Figs 2 and 3 is that similar correlations are found between SFE and sSFR for both LTGs and ETGs. This is interesting because of the proposal that a more prominent stellar bulge in a galaxy stabilizes the gas in the surrounding disc, stopping it collapse to form stars, thus reducing the value of the SFE (Martig et al. 2009).

Figs 2 and 3 contain two pieces of evidence against this idea. The first is that very similar relationships are seen for LTGs and ETGs. If the growth of a bulge is the only process that stops stars forming, we would not expect to see a relationship for galaxies without prominent bulges. The galaxies in our redefined LTG class with the most prominent bulges are the Sc galaxies, but even for these the ratio of the near-infrared luminosity of the bulge to the 


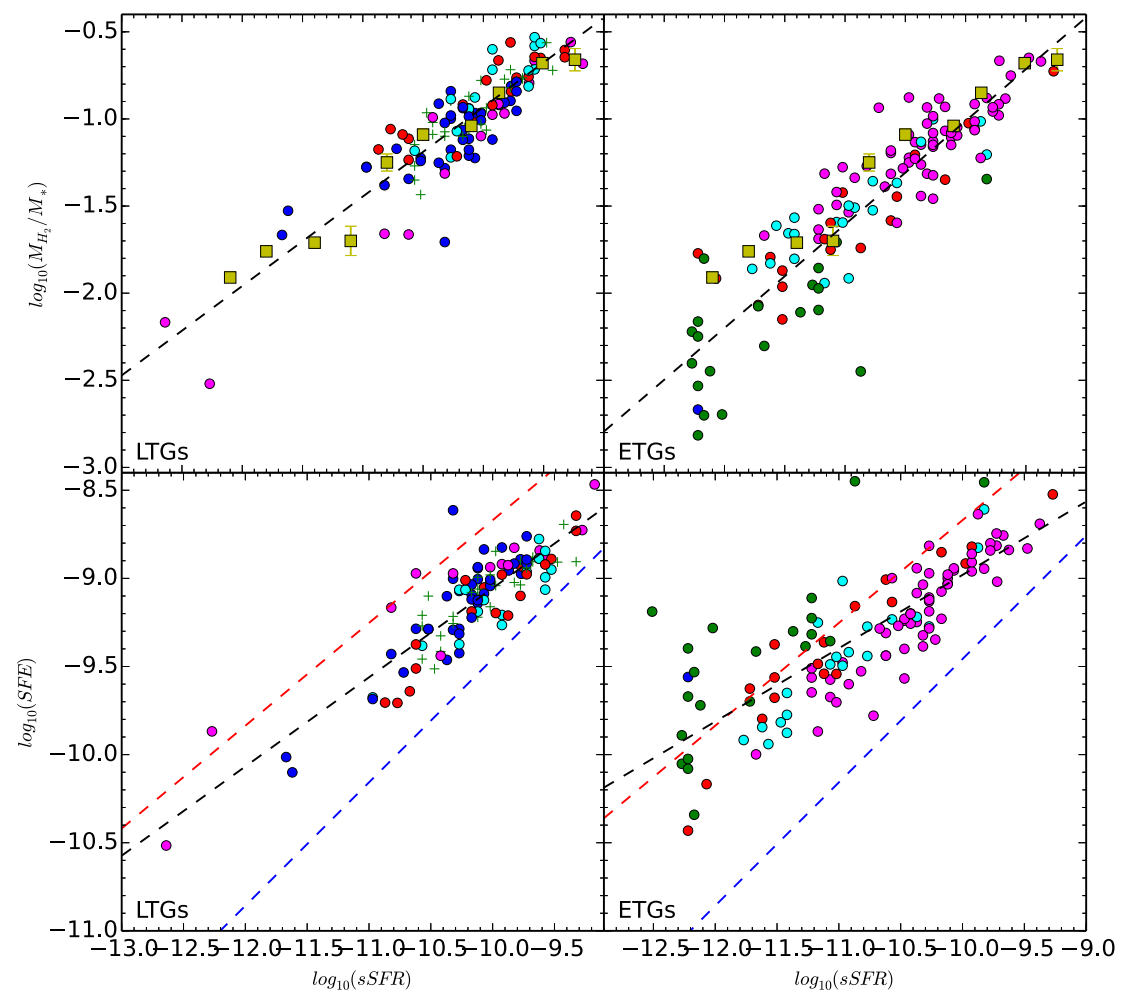

Figure 2. The two top panels show the ratio of gas mass to stellar mass plotted against specific star formation rate (sSFR), the two bottom panels star formation efficiecy (SFE) plotted against sSFR. The gas masses have been calculated using equation (1). The two left-hand panels are for LTGs, which are here defined as Sc galaxies and morphological types that are later along the Hubble sequence. The two right-hand panels show ETGs, here defined as morphological types on the Hubble sequence between, and including, Sbc and E. In the left-hand panels, the colour key is as follows: blue - Sc; green - Scd; red - Sd; cyan - Sm and $\mathrm{Sdm}$; magenta - I, I0 and Im. In the right-hand panels, the colour key is as follows: blue - E and ES0; green - S0 and S0a; red - Sa; cyan - Sab; magenta - Sb and Sbc. The beige squares in the top panels show the average value of gas mass divided by stellar mass for the galaxies in the xCOLD GASS survey (table 6 of Saintonge et al. 2017). The dashed black lines in the panels show the straight lines that best fit the data, the equations for which are given in Table 1. The dashed red lines shows the relationship found at zero redshift between SFE and sSFR by Scoville et al. (2017). The dashed blue lines show the approximate relationship found between SFE and sSFR for the xCOLD GASS survey by Saintonge et al. (2017).

total near-infrared luminosity of the galaxy itself, which should be similar to the bulge-to-total mass ratio, is only $\simeq 10$ percent (Laurikainen et al. 2007). The second piece of evidence is that even within a single morphological class, all of which should have a similar bulge-to-disc ratio, we see the same relationship between SFE and sSFR. The dark blue points in the LTG plots represent $\mathrm{Sc}$ galaxies and the magenta points in the ETG plots represent $\mathrm{Sb}$ and Sbc galaxies; within both classes there is a strong correlation between SFE and SSFR.

Our first conclusion is therefore that the growth of a stellar bulge, and the consequent stabilization of the gas in the disc, is not enough to explain the reduction in the SFE seen in galaxies that have low values of sSFR. There must be some other process at work.

Bluck et al. (2014) have looked at the importance of four different parameters for predicting that a galaxy has a low value of sSFR: stellar mass, halo mass, bulge-to-disc ratio, and bulge mass. They find that the one that is most important is bulge mass. Their conclusion that the bulge-to-disc ratio is comparatively unimportant is consistent with the lack of a strong correlation between Hubble type, which of course depends strongly on bulge-to-disc ratio (Laurikainen et al. 2007), and sSFR in the HRS (Eales et al. 2017; see also Figs 2 and 3). It also suggests that bulge-to-disc ratio cannot be the property that explains the relationship between SFE and SSFR, since if it were we would expect to see strong correlations between bulge-to-disc ratio and both SFE and sSFR. Thus, their work generally supports our conclusion that the disc-stablization idea is not enough to explain the relationship between SFE and sSFR.

Is there any evidence for the disc-stablization hypothesis in the HRS? Both Figs 2 and 3 do suggest that the spread in SFE is larger for ETGs than LTGs, and it is the galaxies with the earliest morphological types that have the lowest values of SFE. Fig. 4, in which we have plotted histograms of SFE for galaxies in different morphological classes, shows this more clearly. For this figure, we have used the values of SFE calculated from the molecular gas masses estimated using equation (1). There is a gradual drop in SFE along the Hubble sequence from later to earlier morphological types, but there seems to be a particularly large jump down in SFE between the fifth and sixth (from the top) panels, at the boundary between $\mathrm{Sb}$ and $\mathrm{Sab}$. We have tested the significance of this fall by treating all the galaxies in the top five histograms as one sample and all the galaxies in the bottom three histograms as a second sample, and then applying the Kolmogorov-Smirnov two-sample test. We find that the Kolmogorov-Smirnov statistic has a value of 0.428 with a formal significance of $4 \times 10^{-8}$.

This agrees with the results of Saintonge et al. (2012). Although they did not have morphological classifications for the galaxies in their sample, they showed that galaxies with low values of SFE also tend to have high values for the SDSS concentration index and central stellar surface density, both of which are generally higher for 


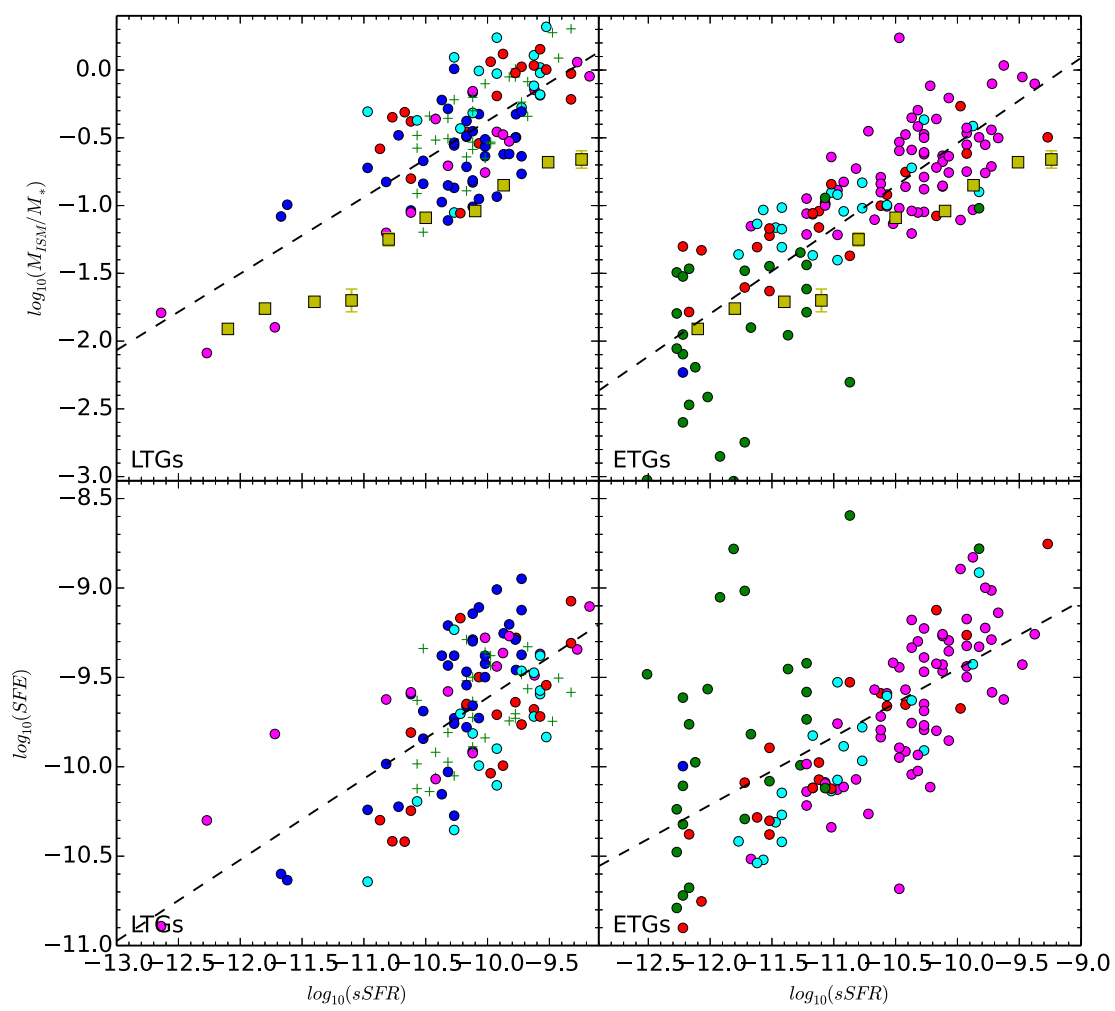

Figure 3. The same as Fig. 2 except that the gas masses have been estimated using equation (2).

Table 1. Relationship between SFE and SSFR.

\begin{tabular}{lcccccc}
\hline Relationship & Morphologies & ISM method & A & B & Spearman & Reference \\
\hline$M_{\text {gas }} / M_{*}$ versus sSFR & LTGs & CO & 0.51 & 4.20 & 0.858 & This paper \\
$M_{\text {gas }} / M_{*}$ versus sSFR & ETGs & CO & 0.59 & 4.93 & 0.840 & This paper \\
$M_{\text {gas }} / M_{*}$ versus sSFR & LTGs & MAGPHYS & 0.56 & 5.27 & 0.630 & This paper \\
$M_{\text {gas }} / M_{*}$ versus sSFR & ETGs & MAGPHYS & 0.63 & 5.76 & 0.825 & This paper \\
SFE versus sSFR & LTGs & CO & 0.51 & -4.00 & 0.822 & This paper \\
SFE versus sSFR & ETGs & CO & 0.42 & -4.82 & 0.840 & This paper \\
SFE versus sSFR & LTGs & MAGPHYS & 0.45 & -5.08 & 0.552 & This paper \\
SFE versus sSFR & ETGs & MAGPHYS & 0.38 & -5.65 & 0.658 & This paper \\
SFE versus sSFR & All & $\ldots$ & 0.58 & -2.84 & $\ldots$ & Saintonge et al. (2017) \\
SFE versus sSFR & All & $\ldots$ & 0.70 & -2.46 & $\ldots$ & Scoville et al. (2017) \\
\hline
\end{tabular}

Table 2. Fits of equations (3) and (4).

\begin{tabular}{lcccc}
\hline Equation & Morphologies & \multicolumn{1}{c}{$\log (\mathrm{A})$} & $\mathrm{B}$ & $\mathrm{C}$ \\
\hline 3 & LTGs & $9.04 \pm 0.01$ & $0.50 \pm 0.01$ & $0.96 \pm 0.01$ \\
3 & ETGs & $8.97 \pm 0.01$ & $0.57 \pm 0.01$ & $0.95 \pm 0.01$ \\
4 & LTGs & $-0.038 \pm 0.004$ & $0.507 \pm 0.006$ & $0.029 \pm 0.006$ \\
4 & ETGs & $0.067 \pm 0.003$ & $0.486 \pm 0.004$ & $0.031 \pm 0.005$ \\
\hline
\end{tabular}

bulge-dominated galaxies. For completeness, since Saintonge et al. also looked at the effect of bars on SFE, Fig. 5 shows histograms of SFE for galaxies with bars and galaxies without bars in the HRS. In the figure, we have lumped together galaxies with strong bars (de Vaucouleurs type SB) and with weak bars (de Vaucouleurs type $\mathrm{SAB}$ ), but it makes little difference if we only put galaxies with strong bars in the barred class. There is a significant difference (Kolmogorov-Smirnov statistic of 0.23 , probability under null hypothesis of identical populations of 0.0018) but it is not a large one. The median $\log _{10}$ (SFE) for the barred galaxies is -9.06 and for the galaxies without bars it is -9.25 . This also agrees well with the results of Saintonge et al. (2012) who also found a significant but small difference between the SFEs of galaxies with and without bars.

We emphasize that the difference in SFE seen in Fig. 4 between the ETGs and LTGs does not prove that it is the effect of the bulge in stabilizing the disc that is responsible for the reduction in SFE. It is possible that the apparent relationship between SFE and morphological class is the result of two separate relationships: a relationship between sSFR and morphological class caused by some unknown process (perhaps bulges formed more readily at earlier cosmic epochs, so galaxies in which most of the stars formed earlier, and thus today have lower values of sSFR, have more prominent bulges) and a relationship between SFE and sSFR caused by the other process for reducing SFE that we have argued must exist. 


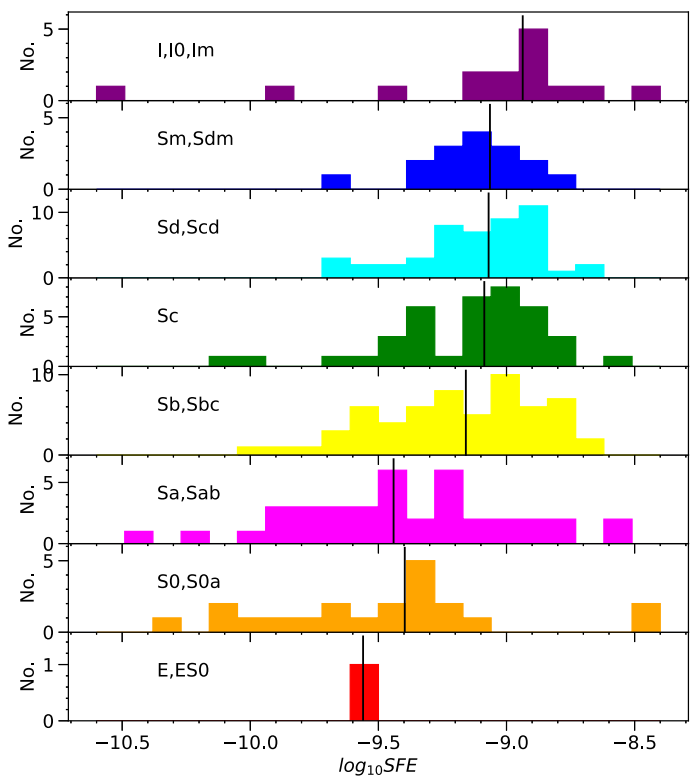

Figure 4. Histograms of star formation efficiency for different morphological classes, which are given by the label in each panel. The star formation efficiency has been calculated using the masses of molecular gas estimated using equation (1). The vertical line is the median star formation efficiency for each morphological class.

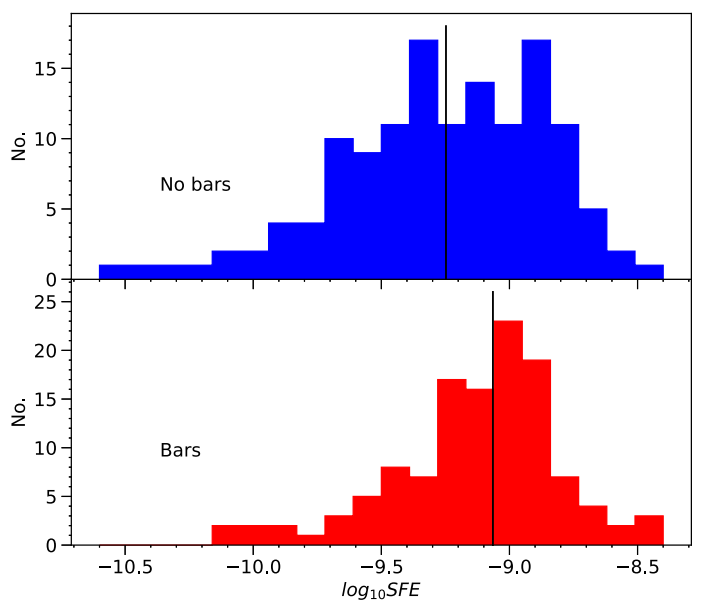

Figure 5. Histograms of star formation efficiency for galaxies without bars (top) and with bars (bottom). In this figure, the barred galaxies include galaxies with de Vaucouleurs morphological classes SB and SAB. The star formation efficiency has been calculated using the masses of molecular gas estimated using equation (1). The vertical line is the median star formation efficiency for each class.

The strong result of this paper is that there must be some other process besides the disc-stabilizing effect of a bulge that must be reducing the SFEs in galaxies, although the observations are consistent with the idea that the disc-stabilizing process is also occurring. As to what this other process is, we do not know. One speculative idea comes from considering the role of turbulence in determining the SFE. Increased turbulence seems to be associated with an increase in the SFE of a galaxy, probably because the increase in turbulence leads to an increase in the fraction of gas that is in a very dense phase (Papadopoulos \& Geach 2012). Turbulence is injected into a galaxy by both the accretion of gas and feedback from star formation (Krumholz et al. 2018), so the current velocity field in a galaxy is the result of the history of both processes. A plausible, but clearly very speculative, explanation of the low values of SFE for galaxies with low values of SSFR is that the main star formation phase of these galaxies will have been further back in the past, giving more time for the velocity field, stirred up by the gas accretion and feedback during the star formation phase, to have quietened down. A simple test of this hypothesis would be to investigate whether any of the statistical measures of a galaxy's velocity field depend on SSFR for galaxies in the Universe today, a test well within the capabilities of the Atacama Large Millimetre Array.

\section{CONCLUSIONS}

In this paper, we have used the $H R S$ to make a direct test of the hypothesis that the growth of a stellar bulge leads to a reduction in the SFE (or conversely an increase in the gas-depletion timescale) as the result of the stabilization of the gaseous disc by the gravitational field of the bulge. We find a strong correlation between SFE and sSFR in galaxies without prominent bulges and in galaxies of the same morphological type, showing that there must be some other process besides the growth of a bulge that reduces the SFE in galaxies.

However, we also find that galaxies with more prominent bulges do tend to have lower values of SFE, confirming the conclusion of Saintonge et al. (2012), who used a more indirect method that did not use morphological classifications. Confirming another result of Saintonge et al. (2012), we also find a significant but small difference between the SFEs in galaxies with and without bars, in the sense that galaxies with bars have slightly higher values of SFE.

Our strong conclusion is that there must be some other process beyond the disc-stabilizing effect of a bulge that is responsible for the reduction of the SFE in galaxies. Our weaker conclusion is that the difference in SFE between different morphological classes is at least consistent with the hypothesis that the growth of a bulge stabilizes the gas in the disc, thus reducing the SFE.

\section{ACKNOWLEDGEMENTS}

We thank Asa Bluck for many useful discussions. We thank Asa Bluck and Frederic Bournaud for comments on the manuscript that significantly improved it. SAE thanks Clare Hall in Cambridge for a Visiting Fellowship, and the college, the Kavli Institute and the Institute of Astronomy for making his visit to Cambridge an enjoyable and stimulating one. SAE acknowledges funding from the UK Science and Technology Facilities Council consolidated grant ST/K000926/1.

\section{REFERENCES}

Abdo A. A. et al., 2010, ApJ, 710, 133

Baldry I. K. et al., 2012, MNRAS, 441, 2440

Baum W. A., 1959, PASP, 71, 106

Bell E. R., McIntosh D. H., Katz N., Weinberg M. D., 2003, ApJS, 149, 289

Bluck A., Mendel T., Ellison S., Moreno J., Simard L. , Patton D., Starkenburg E., 2014, MNRAS, 441, 599

Boselli A. et al., 2010, PASP, 122, 261

Boselli A., Cortese L., Boquien M., 2014, A\&A, 564, 65

Bruzual G., Charlot S., 2003, MNRAS, 344, 1000

Cappellari M. et al., 2013, MNRAS, 432, 1862

Charlot S., Fall S. M., 2000, ApJ, 539, 718

Ciesla L. et al., 2012, A\&A, 543, A161

Clark C. J. R., Schofield S. P., Gomez H. L., Davies J. I., 2016, MNRAS, 459,1646 
Cortese L., 2012, A\&A, 543, 132

Cortese L. et al., 2014, MNRAS, 440, 942

Cortese L. et al., 2016, MNRAS, 463, 170

da Cunha E., Charlot S., Elbaz D., 2008, MNRAS, 388, 1595

Davies L. J. M. et al., 2016, MNRAS, 461, 458

De Vis P. et al., 2017, MNRAS, 464, 4680

Eales S. et al., 2012, ApJ, 761, 168

Eales S., de Vis P., Smith M. W. L., Appah K., Ciesla L., Duffield C., Schofield S., 2017, MNRAS, 465, 3125

Eales S. et al., 2018a, MNRAS, 473, 3507

Eales S. et al., 2018b, MNRAS, 481, 1183

Genzel R. et al., 2014, ApJ, 785, 75

Groves B. A. et al., 2015, ApJ, 799, 96

Hayward C. C., Smith D. J. B., 2015, MNRAS, 446, 1512

Hughes T. M., Cortese L., Boselli A., Gavazzi G., Davies J. I., 2013, A\&A, $550, \mathrm{~A} 115$

James A., Dunne L., Eales S. A., Edmunds M., 2002, MNRAS, 335, 753

Janowiecki S., Cortese L., Catinella B., Goodwin A. J., 2018, MNRAS, 476, $1390 \mathrm{ng}$

Kennicutt R. C., Evans N. J., 2012, ARAA, 50, 531

Krumholz M. R., Burkhart B., Forbes J. C., Crocker R. M., 2018, MNRAS, 477,2716

Laurikainen E., Salo H., Buta R., Knapen J. H., 2007, MNRAS, 381, 401

Magdis G. E. et al., 2012, ApJ, 760, 6

Martig M., Bournaud F., Teyssier R., Dekel A., 2009, ApJ, 707, 250

Papadopoulos P. P., Geach J. E., 2012, ApJ, 757, 157

Pineda J. L., Langer W. D., Velusamy T., Goldsmith P. F., 2013, A\&A, 554, A103

Planck Collaboration XIX, 2011, A\&A, 536, A19

Planck Collaboration, 2014, A\&A, 571, 16
Popescu C. C., Tuffs R. J., Dopita M. A., Fischera J., Kylafis N. D., Madore B. F., 2011, A\&A, 527, 109

Rémy-Ruyer A. et al., 2014, A\&A, 563, A31

Saintonge A. et al., 2011, MNRAS, 415, 61

Saintonge A. et al., 2012, ApJ, 758, 73

Saintonge A. et al., 2017, ApJS, 233, 22

Sandstrom K. M. et al., 2013, ApJ, 777, 5

Scoville N. et al., 2014, ApJ, 783, 84

Scoville N. et al., 2017, ApJ, 837, 150

Smith M. W. L. et al., 2012, ApJ, 748, 123

Strateva, I. et al., 2001, AJ, 122, 1861

Tacconi L. J. et al., 2018, ApJ, 853, 179

\section{APPENDIX A: ARE RELATIONSHIPS BETWEEN SFE AND SSFR CAUSED BY EXPERIMENTAL ERRORS}

Both SFE and sSFR are ratios with SFR as the numerator in the ratio. Therefore, errors in the estimates of the SFR could potentially generate spurious relationships between SFE and sSFR. We have tested this possibility using the following simple Monte Carlo procedure.

In this test, we used the molecular gas masses of Fig. 2 rather than the MAGPHYS gas masses of Fig. 3. The simulation is based on the assumption that all galaxies in a morphological class (LTGs or ETGs) have the same value of SFE, which we take as our mean estimate of the SFE for the galaxies in that class. We then start with the MAGPHYS stellar mass and our estimate of the gas mass of each galaxy and use the assumed SFE to calculate a SFR for that galaxy. In the absence of errors, there would then be no correlation

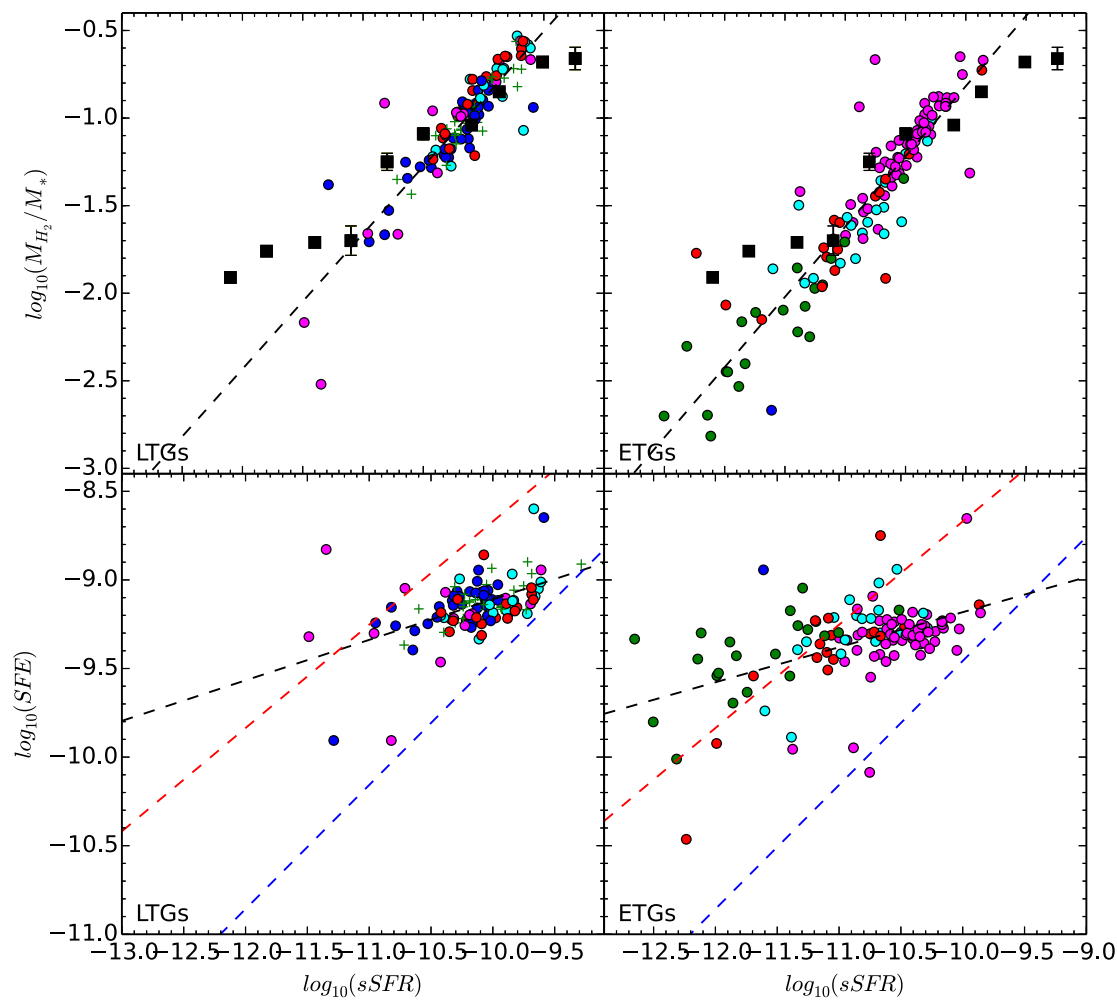

Figure A1. The same as Fig. 2 except that the star formation rates have been generated using the Monte Carlo method described in Appendix A, which is based on the assumption that all galaxies in a morphological class (LTGs or ETGs) have the same value of SFE. We have produced this figure by making the assumption that the errors in the star formation rate are those given by MAGPHYS. The equations for the best-fitting lines (the dashed black lines) are given in Table A1. 
Table A1. Monte Carlo Results.

\begin{tabular}{lcccrc}
\hline Relationship & Morphologies & Errors & A & B & Spearman \\
\hline$M_{\text {gas }} / M_{*}$ versus sSFR & LTGs & MAGPHYS & 0.77 & 6.82 & 0.884 \\
$M_{\text {gas }} / M_{*}$ versus sSFR & ETGs & MAGPHYS & 0.80 & 7.20 & 0.921 \\
SFE versus sSFR & LTGs & MAGPHYS & 0.23 & -6.82 & 0.470 \\
SFE versus sSFR & ETGs & MAGPHYS & 0.20 & -7.20 & 0.423 \\
$M_{\text {gas }} / M_{*}$ versus sSFR & LTGs & MAGPHYS $\times 3$ & 0.37 & 2.74 & 0.616 \\
$M_{\text {gas }} / M_{*}$ versus sSFR & ETGs & MAGPHYS $\times 3$ & 0.56 & 4.62 & 0.800 \\
SFE versus sSFR & LTGs & MAGPHYS $\times 3$ & 0.63 & -2.74 & 0.689 \\
SFE versus sSFR & ETGs & MAGPHYS $\times 3$ & 0.44 & -4.62 & 0.476 \\
\hline
\end{tabular}

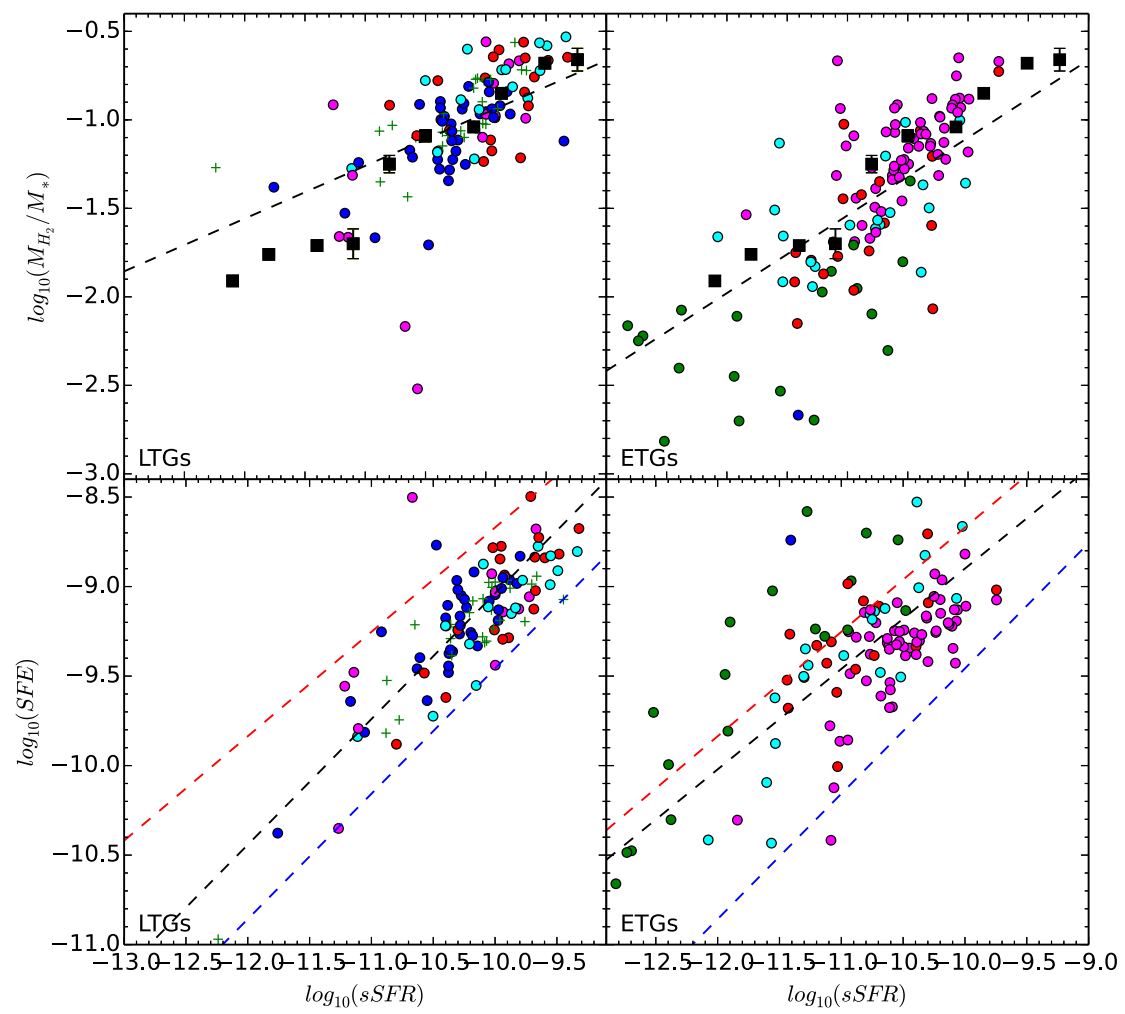

Figure A2. The same as Fig. 2 except that the SFRs have been generated using the Monte Carlo method described in Appendix A, which is based on the assumption that all galaxies in a morphological class (LTGs or ETGs) have the same value of SFE. We have produced this figure by making the assumption that the errors in $\log _{10}$ SFR are three times those given by MAGPHYS. The equations for the best-fitting lines (the dashed black lines) are given in Table A1.

between SFE and sSFR. We generate an error for each galaxy using the errors on $\log _{10}$ SFR given by MAGPHYS. Fig. A1 shows the result, which should be compared with Fig. 2.

The figure shows that a correlation between SFE and sSFR has been generated by the errors in the SFR. The dashed black lines in the figure show the straight lines that are the best fit to the data, and Table A1 lists the equations for these lines and the values of the Spearman correlation coefficient. The errors clearly produce a significant correlation but the slope of the relationship between SFE and SSFR is much smaller than the slope of the relationship seen in Fig. 2.

It is possible, of course, that the errors in the SFR given by MAGPHYS are underestimates. We have investigated this possibility by making the assumption that the true errors in our values of $\log _{10}$ SFR are three times greater than those given by MAGPHYS. Fig. A2 shows the result. This time there is a strong correlation between SFE and sSFR, as strong as that seen in Fig. 2. However, the relationship in the top two panels between $\mathrm{M}_{\mathrm{gas}} / \mathrm{M}_{*}$ and sSFR is now weaker than those in the top two panels of Fig. 2. We therefore conclude that that the relationships shown in Fig. 2 are not the result of errors in the SFR generating correlated errors in SFE and SSFR.

\section{APPENDIX B: APPLICATION OF THE METROPOLIS-HASTINGS TECHNIQUE TO THE DATA}

We fitted equations 3 and 4 to the data by minimizing chi-squared. We fitted equation (3) by minimizing chi-squared on the assumption that the errors in $\log _{10} M_{\text {gas }}$ are 0.2 . We fitted equation (4) using the errors in $\log _{10}$ SFR produced by MAGPHYS. We sampled the posterior probabilty distribution of $\mathrm{A}, \mathrm{B}$, and $\mathrm{C}$ using the Metropolis-Hasting algorithm, with 500000 points and a burn-in phase of 2000 points. The probability distributions and covariance plots for $\mathrm{A}, \mathrm{B}$, and $\mathrm{C}$ are shown for LTGs for the two equations in Figs B1 and B2. 

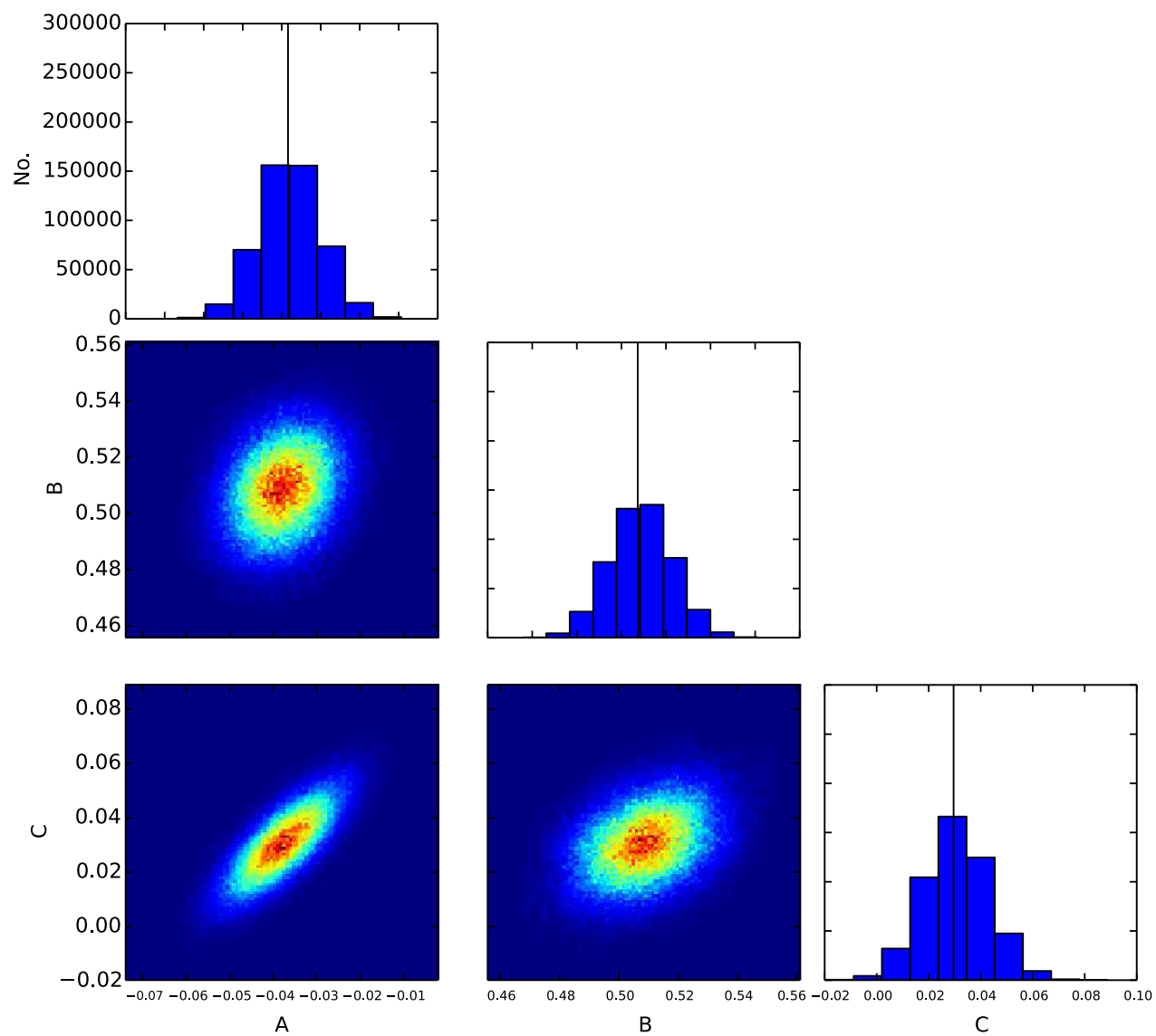

Figure B1. The results of fitting equation (3) to the data for the late-type galaxies (as defined in Section 3). The histograms show the probability distributions for the three parameters, with the vertical lines showing the mean values. The other panels show covariance plots. 

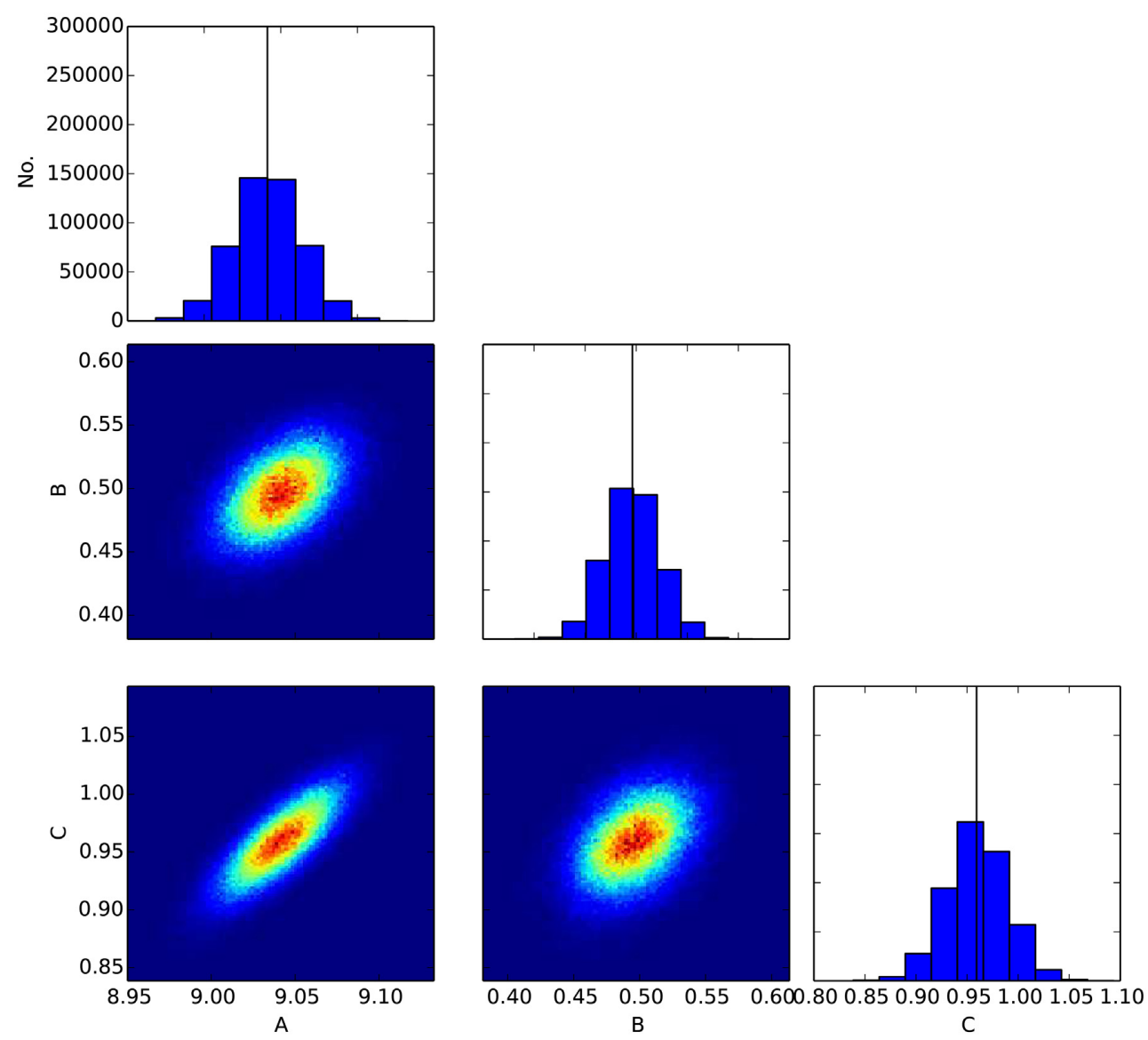

Figure B2. The results of fitting equation (4) to the data for the late-type galaxies (as defined in Section 3). The histograms show the probability distributions for the three parameters, with the vertical lines showing the mean values. The other panels show covariance plots.

This paper has been typeset from a $\mathrm{T}_{\mathrm{E}} \mathrm{X} / \mathrm{L} \mathrm{T} \mathrm{E} \mathrm{X}$ file prepared by the author. 